\title{
Freezing of Gait Detection Considering Leaky Wave Cable
}

\author{
Xiaodong Yang, Senior Member, IEEE, Syed Aziz Shah, Aifeng Ren, Nan Zhao, Zhiya Zhang, Dou Fan, \\ Jianxun Zhao, Weigang Wang, Masood Ur-Rehman, Senior Member, IEEE
}

\begin{abstract}
A novel study on monitoring and analysis of debilitating condition of patients suffering from neurological disorder is presented. Parkinson's disease is characterized by limited motor-ability of a patient. Freezing-of-gait is a major non-motor condition among ageing patients and its evaluation can reduce the chances of any secondary disorders. In this work, amplitude and phase information of the radio signals observed for a fixed period of time are used to differentiate the motor and non-motor symptoms. The amplitude information is classified using Support Vector Machine while linear transformation is applied to obtain sanitized phase information for detection. The proposed method is very handy with minimum deployment overhead. The analysis shows that this method also offers a high accuracy level of around $99 \%$ based on the observation of a number of patients. These features make it an attractive solution for real-time patient monitoring systems.
\end{abstract}

Index Terms - Radio Propagation, Antennas, Leaky Wave Cable (LWC), Parkinson's disease, Freezing-of-Gait.

\section{INTRODUCTION}

Parkinson's disease (PD) is a neurodegenerative disorder that has affected over 16.1 million people [1]. PD causes muscular and motor impairments that increase with age. It eventuates in rigidity, tremors, slow movement and postural instability in a person [2]. The hallmark signs include freezing and arching of posture. A particular disabling aspect of the Parkinsonian gait especially in advanced stages is freezing episodes or freezing-of-gait (FOG). FOG is defined as the inability of feet to carry a forward progression. The FOG

The work was supported in part by the National Natural Science Foundation of China (No. 61671349, 61301175, 61601338), China Postdoctoral Science Foundation Funded Project (No. 2018T111023), International Scientific and Technological Cooperation and Exchange Projects in Shaanxi Province (No. 2017KW-005), Fundamental Research Funds for the Central Universities (No. JB180205).

Xiaodong Yang, Aifeng Ren, Nan Zhao, Zhiya Zhang, Dou Fan, Jianxun Zhao are with School of Electronic Engineering, Xidian University, Xi'an, Shaanxi, 710071, China.

S. A. Shah is with the School of International Education, Xidian University, Xi'an, Shaanxi, 710071, China; and also with School of Engineering, University of Glasgow, G12 8QQ, United Kingdom.

Weigang Wang is with Northwest Women's and Children's Hospital of Xi'an Jiaotong University, Xi'an, Shaanxi, 710061, China.

Masood Ur Rehman is with the School of Computer Science and Electronic Engineering at University of Essex, Colchester CO4 3SQ, UK. affects the movement of the patient along with arms and eyelids while walking. It gets triggered when the subject tries to turn described as motor blocks. The patient has to take several steps instead of pivoting on one foot while changing direction resulting in a freeze and experiencing a sudden and momentary loss of movement. The patient undergoes hesitation in walking or moving the feet for a certain time period. Fear of falling and losing control adds to the loss of motor skills. The FOG and posture instability are major causes of such patient's falls and result in high risk of hip fractures [2]. The PD thus is different from other neurodegenerative diseases that affect autonomic nervous system [2].

Several behavioral methods have been presented by researchers to reduce freezing effects. These methods include walking to a beat, slide a foot forward, marching or swinging the arms, and straight walk to improve balance [3-9]. Such external cues are commonly considered effective in lessening FOG symptoms in the patients [3]. To measure the effectiveness of such methods, a measurement system independent of the PD patient's attention is essential. Open literature presents different systems designed to monitor and assess the PD and FOG [4]-[5]. In these systems, sensors and the associated technology should be able to deliver accurate data during gait analysis test. To improve accuracy, several systems using wireless body sensor networks have been designed to monitor the FOG providing an objective and quantitative assessment [6]-[9]. The readings need to be differentiated since the systems are worn on patient's body.

With the development of communication standards, applications based on reflections and interference of electromagnetic waves eliminate need of wearing the sensors. The idea is that a person's movement acts as an obstacle to the wave propagation and modulates radio signals due to temporal changes. These changes can be observed effectively at the receiving end of the communication system. The pattern of the received radio signal is then processed to identify certain postures and movements of the human subject. Typical applications of such systems include patients and children monitoring, human intrusion detection and gesture recognition. $\mathrm{Pu}$ et al. [10] and $\mathrm{Xu}$ et al. [11] have used non-invasive methods to extract motion activity from radio signals and track gestures and localize body motions. The key advantage of such wireless systems is that they eliminate the need of a sensor to be body-worn and hence, improves the accuracy over short durations of time.

In this study, we have employed the same phenomenon to evaluate the FOG conditions in patients suffering from the PD. 
Our investigation on the interference of a person's presence on the radio signals demonstrates that the normal walking pattern and the FOG conditions induce different changes in the radio signal patterns, which in turn result in detection of PD patient's FOG [12]. The proposed method offers a non-invasive detection of the FOG through physical layer channel information employing a leaky wave cable (LWC). The channel information offers fine-grained channel description at the granularity of OFDM subcarriers [13]. Use of a LWC on the receiving end can accommodate multiple patients located in different rooms in a hospital environment. To identify the normal and freezing-of-gait conditions, the amplitude information is classified using support vector machine (SVM) while linear transformation is applied on measured channel phase information to extract useful information out of the extremely random raw data.

Following this introduction, the paper is organized in four sections. Section II describes the data acquisition process. Section III details the measurement test-bed. Results are analyzed in Section IV and conclusions are drawn in Section V.

\section{DATA ACQUISITION}

The proposed system uses Cards to record channel packets per subcarrier between transmitter and receiver. The received signal is $Y=H \times X$ where $H$ represents channel frequency response (CFR), $X$ and $Y$ are transmitted and received signals, respectively. The measured data for 30 OFDM subcarriers contains the CFR values and can be expressed as:

$$
\mathbf{H}=\left(h_{1}, h_{2}, h_{3}, \ldots, h_{30}\right)
$$

A packet carries the raw amplitude information and random phase data as follows:

$$
\mathbf{H}(n)=\mid \mathbf{H}(n) \| e^{<H(n)},
$$

in this expression, $\mathbf{H}(n)$ is the data for subcarrier number $n$, where $n \in[1$ to 30$] .\|\mathbf{H}(n)\|$ is the raw amplitude information and $\angle H(n)$ denotes the random phase data.

The time history for FOG detection is obtained by examining the channel information amplitude information for $x$ number of measurements. It can be expressed as:

$$
\mathrm{CFR}_{\text {time_history }}=\left(\mathbf{H}_{1}, \mathbf{H}_{2}, \mathbf{H}_{3}, \ldots, \mathbf{H}_{x}\right) \text {. }
$$

\section{A. Classification Method}

Classification procedure involves assigning a particular object to certain number of classes. A training data set is used to train the classifier where each object's class is known. Once a classifier is trained, it can be used to a new object to its corresponding class; while in test mode the classifier is assigned to the test dataset. The overall performance of the classifier is evaluated by analyzing and comparing the classifications made using test dataset. In this study, we have classified the amplitude information obtained for walking and freezing-of-gait using support vector machine (SVM). The SVM is a sophisticated learning technique that primarily distinguishes between two classification problems. A hyper-plane is used as the decision boundary to separate two datasets and can be defined as:

$$
w^{T} x+\beta_{0}=0
$$

here $w$ is the weight vector, $\beta_{0}$ is the bias and $x$ represents input vector.

The optimal hyper-plane can be obtained in various ways by scaling the values of $w$ and $\beta_{0}$. The data points nearest to the hyper-plane are known as support vectors [14]. The support vectors for each class can be expressed as:

$$
\begin{aligned}
& w^{T} x+\beta_{0}=+1 \text { for } d_{i}=+1(\text { Class A), } \\
& w^{T} x+\beta_{0}=-1 \text { for } d_{i}=-1 \quad(\text { Class B }) .
\end{aligned}
$$

The optimal hyper-plane for optimization problem of training dataset can be described as:

$$
\begin{array}{r}
\min (\vartheta)=\frac{1}{2} w^{T} w, \text { such that } d i=\left(w T+\beta_{0}\right) \geq 1, \quad \text { (7) } \\
\text { for } \mathrm{i}=1,2,3, \ldots
\end{array}
$$

The final decision function can be formulated as:

$$
f(x)=\operatorname{sign}\left[\sum_{i=1}^{N} \alpha_{o, i}\left(x^{T} x_{i}\right)+b\right],
$$

where $x$ is the input vector, $N$ represents the total number of support vectors. The parameter $\alpha_{o, i}$ is used to determine the support vectors for $x$.

The SVM is a powerful algorithm and is able to map the nonlinearly separable classes into a high-dimensional feature space where the classes become linearly separable using a nonlinear kernel function [14]-[15]. Any appropriate kernel function can be selected which makes this algorithm computationally efficient [14]. In this study, we have used classical Pearson kernel function due to its well-known features and high accuracy.

\section{B. Phase sanitization using linear transformation}

The phase information is extremely random over all feasible fields due to unsynchronized timing and random noise between transceiver pair. It is therefore, cannot be used for detecting FOG episodes. In order to use phase information for effective detection, linear transformation on random phase data is performed to mitigate the impact of random noise and phase offset [16]. 


\section{MEASUREMENT TESTBED}

The proposed FOG detection system employs a leaky wave cable (LWC) through physical layer channel information to differentiate between the FOG and the normal walking pattern. We explain the reason for using a LWC and describe the experimental measurements in this section.

\section{A. Rationality of using Leaky Wave Cable}

One of the main reasons for using LWC instead of omni-directional antenna is to get an improved performance in terms of directivity and ease of deployment. It is widely used for its reduced Inter-Symbol Inference (ISI) and interference of channelizing wireless signals where conventional antennas fail. The LWC consists of periodic slots that have a highly directive radiation pattern. The directivity depends on the RF signal wavelength, propagation angles, periodicity of the slots and relative permittivity of the material. The periodicity of the LWC slots (as shown in Figure 1) makes the cable very efficient in radio signal reception. Wireless signal is transmitted and received through these zigzag slots.
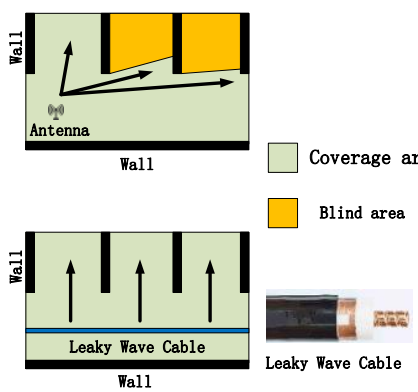

Figure 1 - Conventional antenna generates blind zones while LWC provides wider service area.

Moreover, in comparison to using a conventional antenna, wireless access systems with LWC are robust. Installation of the access point unit and overloads of the handover mechanism within one cable segment is minimal [17].

Considering radiation mode of the LWC, energy transmission is in radial form and the zigzag slots act like an array antenna. The radio signal is transmitted and received along the cable.

For a periodic structure considering radiating mode, the electric field around the LWC can be written as [18]:

$$
E(d, z)=-\frac{j}{4} \sum_{p=-\infty}^{\infty} \alpha_{p} M H_{1}^{2}\left(\alpha_{p} d\right) e^{\left(-j k_{p}^{\prime} z\right)}
$$

here $H_{1}^{2}($.$) represents the Hankel function of second order, M$ denotes the magnetic current for each radiating slot, $d$ is the distance along the radial direction and $z$ is the distance along the axis which the LWC is radiating energy. $\alpha_{p}=\sqrt{k_{0}^{2}-k^{\prime 2}}$, $k^{\prime} p=k^{\prime}+\frac{2 \pi p}{T}$, while the terms $\quad k^{\prime}=\frac{2 \pi f \sqrt{\epsilon_{r}}}{c}$ and $k_{0}=\frac{2 \pi f}{c}$ are the wave number for the LWC and free space, respectively. $\epsilon_{r}$ is the permittivity of free space, $T$ represents the prime period of the LWC slots and $c$ is the speed of light.

Figure 1 illustrates the LWC signal acquisition comparison with an antenna in a closed structure. An Omni-directional antenna that has circular coverage area creates shadow zones with structural obstruction. As a result, there are high chances of missing the data. The LWC covers the blind zones, provides seamless data reception and deployment is very handy. Therefore, a single piece of the LWC is used in this work at the receiver side to cover multiple rooms in a hospital environment.

The minimum bending radius of the LWC is $280 \mathrm{~mm}$. Its characteristic impedance is $50 \Omega$ and the capacitance is 76 $\mathrm{pF} / \mathrm{m}$. The transmission attenuation at the working frequency is $10.17 \mathrm{~dB} / 100 \mathrm{~m}$ and the VSWR within the frequency band is less than 1.3. The measurement of loss of signals is based on the method IEC 61196-4 [19], and the capacitance would cause the loss qualitatively.

\section{B. Measurement Testbed}

A FOG monitoring system for single and multiple subjects is presented in Figure 2(a) and Figure 2(b), respectively. We have used a Microwave Sensing Platform (MSP) to collect data. The platform measurement setup is shown in Figure 3. The platform includes spectrum analyzer, vector network analyzer, leaky wave cable, software-defined radio, RF generator, and desktop workstation equipped with network adaptor. The platform can work at a number of frequencies. In our experiments, the RF generator connected with the LWC is used as the transmitter to transmit the RF signal at $2.4 \mathrm{GHz}$, the bandwidth is $20 \mathrm{MHz}$, and the output power of RF generator is set as $-5 \mathrm{dBm}$. The information is sent to desktop workstation equipped with network adaptor for further processing. The data is collected while monitoring the freezing episodes of the PD patients. The change in person's gait impacts the propagation path and the resulting channel information packets received through multiple paths are distinctive. Analyzing the channel information packets over a period of time allows determination of the freezing of gait for individual or multiple subjects in monitoring room(s). The normal and FOG detection is verified for single and multiple cases.

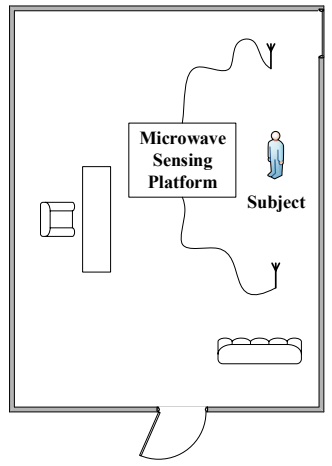

(a) 


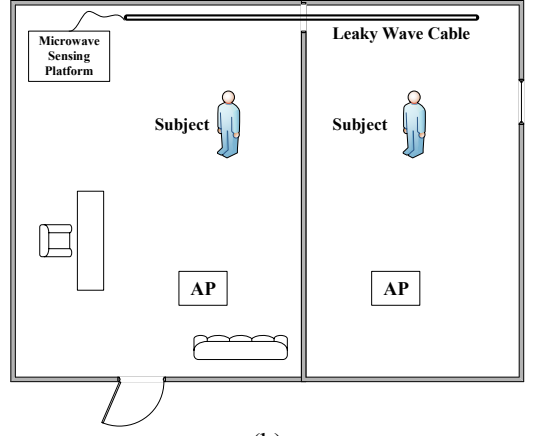

(b)

Figure 2 - (a). Single room setup to monitor FOG for single patient. (b). Two room setup to monitor FOG for multiple patients.

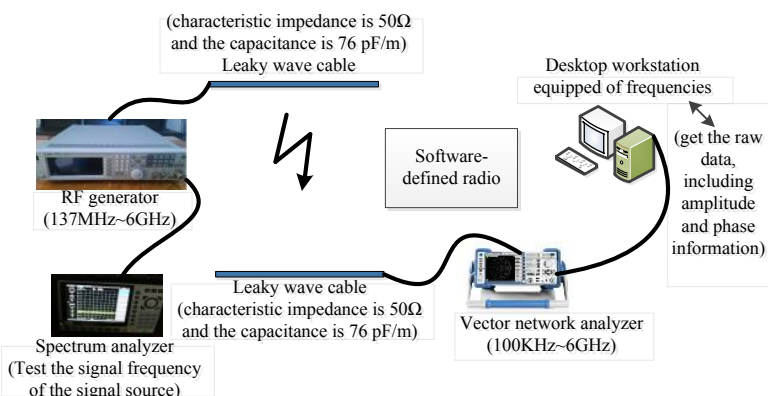

Figure 3 - MSP measurement setup.

It is noticeable that semi-spherical gain antenna will also cover blind zones; our consideration is that when LWC is installed across multiple rooms, the complexity can be decreased. Signal acquisition is done using the LWC receiver and data is formatted as a 30 -subcarrier group in the form of a matrix.

The data signals are received when data packets are forwarded from the LWC. An LWC of 1-meter length is used as the receiving antenna. The other end of the cable is connected to the receiver. The channel information tool-chain is used for data collection and post processing.

Obstacles within wireless range causes the radio waves to fluctuate greatly when physical objects move within wireless range, as a result multiple signal propagation paths are formed. While such effects are often averaged out, when looking at a single average RSS measurement, the individual subcarrier measurements are more likely to change when small movements have altered the multipath environment [20].

The proposed method leverages stationary and motion-induced channel information amplitude fluctuations per channel for a period of time. The communication path observes subtle variation when the subject moves around inside the room. In line-of-sight (LOS) propagation, the transmitter and receiver can send and receive data without any obstruction. However, non-line-of-sight (NLOS) is a type of propagation in which a radio transmission across a path is obstructed by a physical object resulting in reflection, diffraction and scattering. Thus, the received data for different gait conditions will have different channel information values. The system classifies FOG for patients over a time period and the collected data is analyzed to differentiate the two conditions.

\section{Evaluation AND ANALYSES}

The transmitter-receiver pair is kept at a distance of 4 meters. Two scenarios are considered; a single PD patient being observed for the FOG episodes in a single room and two PD patients being observed simultaneously located in two separate rooms to replicate a multiple-patient hospital environment. For the single patient scenario, a large meeting room (15 meters $\times 11.8$ meters in length) is selected at Electronics Science and Technology University as illustrated in Figure 2(a). For the multiple patients' scenario, two test subjects each stationed in the large conference room and the neighboring sitting room are observed as shown in Figure 2(b).

Two dedicated routers operating at distinct frequencies of $2.41 \mathrm{GHz}$ and $2.45 \mathrm{GHz}$ are deployed in each room to avoid co-channel interference. And the transmitter power of these two routers is adaptive. A modified driver is used to record channel information data that pings the access point at 20 packets/second. The wireless link experiences multipath fading depending on the location of transmitter and receiver and the patient's movement.

The raw data is processed in time and frequency domain to extract useful information and identify the FOG. The experiment is repeated for different subjects as presented in Table- 1 and Table-2.

Table I Experimental data for monitoring FOG in individual patient

\begin{tabular}{|l|c|c|c|}
\hline \multicolumn{3}{|c|}{ Experimental data | Monitoring FOG of individual patient } \\
\hline Subject -ID & $\begin{array}{c}\text { Weight } \\
\text { (pound) }\end{array}$ & $\begin{array}{c}\text { Height } \\
\text { (meter) }\end{array}$ & $\begin{array}{c}\text { Speed } \\
\text { (m/s) }\end{array}$ \\
\hline 1 & 187.39 & 1.69 & 0.94 \\
\hline 2 & 174.16 & 1.73 & 1.21 \\
\hline 3 & 158.73 & 1.70 & 1.10 \\
\hline
\end{tabular}

Table II Experimental data for monitoring FOG in multiple patients

Experimental data | Monitoring FOG of multiple patients

\begin{tabular}{|l|l|l|c|}
\hline Subject -ID & $\begin{array}{c}\text { Weight } \\
\text { (pound) }\end{array}$ & $\begin{array}{c}\text { Height } \\
\text { (meter) }\end{array}$ & $\begin{array}{c}\text { Speed } \\
(\mathrm{m} / \mathrm{s})\end{array}$ \\
\hline 1 & 187.39 & 1.69 & 0.89 \\
\hline 2 & 174.16 & 1.73 & 1.31 \\
\hline 3 & 158.73 & 1.70 & 1.05 \\
\hline 4 & 165.34 & 1.73 & 1.25 \\
\hline 5 & 150.3 & 1.67 & 0.90 \\
\hline
\end{tabular}

A change in the channel information is observed when the person is walking in the room.

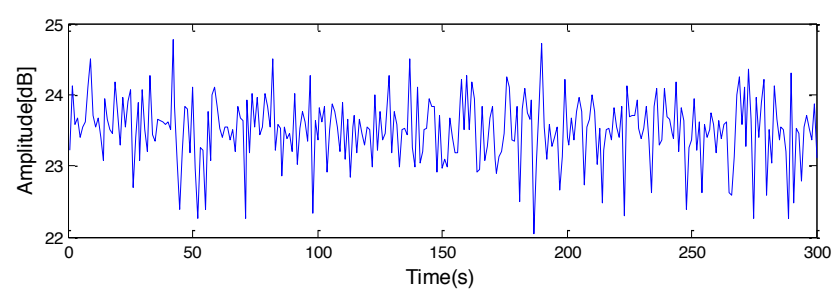

Figure 4 - Time history of amplitude information when no one exists in the experimental room.

Level Cross Rate (LCR) describes how the channel changes with time. $L C R=\sqrt{2 \pi} f_{m} \rho e^{-\rho^{2}}$. Where $\mathrm{f}_{\mathrm{m}}$ is the maximum Doppler shift, which is given by $\mathrm{f}_{\mathrm{m}}=\frac{\mathrm{v}}{\mathrm{c}} \mathrm{f}_{\mathrm{c}}$, and $\mathrm{f}_{\mathrm{c}}$ is the center 
frequency of the transmitter, and $\rho$ is the threshold level which has been normalized to the root mean square (RMS) signal level. Theoretically, there is no Doppler shift when no object moving between the transmitter and receiver, LCR is 0 . However, in the actual experiment, due to the effect of surrounding environment, the fluctuation of the amplitude is $3 \mathrm{~dB}$, as given in Figure 4. The observation of Doppler shift is another computing method that can be useful on the differentiation of the two gait behaviors; a discussion on Doppler shift and LCR in further detail is out of the scope of this work and can serve as a future extension of the study.

\section{A. Scenario I: FOG detection in a single patient}

Channel information is a $30 \times \mathrm{k}$ matrix, where $\mathrm{k}$ represents total number of packets received. Figure 5 shows the CFR and time history for persons' normal and freezing of gait for received packet stream.

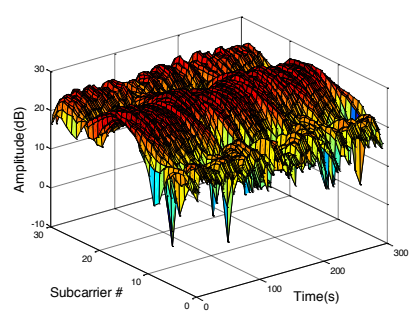

(a). Walking

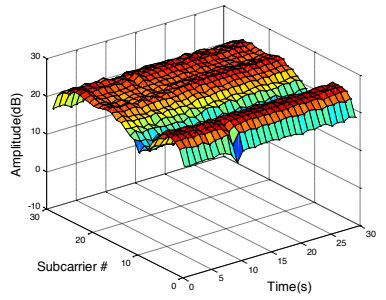

(b). Freezing of gait
Figure 5-(a). Raw CFR traces for subject walking inside the room for 300 seconds.

(b). Raw CFR data for subject experiencing freezing of gait for a period of 30 seconds.

The raw channel frequency traces show great variations due to the person walking across the room for a period of 300 seconds. The stationary CFR values in Figure 5(b) show that the subject was observing freezing for 30 seconds.

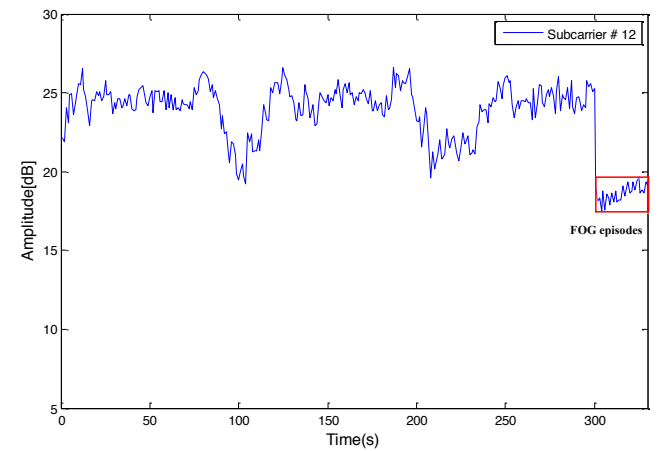

Figure 6 - Time history of amplitude information when person was walking and experiencing freezing episodes

Figure 6 describes variances in power level against time history of subcarrier \# 12 when subject was walking for 300 seconds and suddenly started experiencing freezing episodes for 30 seconds. The time history shows that the experiment was conducted for period of 330 seconds. The limitation in detecting a particular gait based on the amplitude information only is that there are three dips in power level and identifying which of them corresponds to the freezing episode is difficult.
Thus, we use support vector machine to classify walking and freezing of gait by considering the raw amplitude information as shown in Figure 5(a) and Figure 5(b).

Before using SVM classifier, the data is preprocessed to extract features of freezing symptom and normal gait (walking). Feature selection is the essential step for classification. In this work, we considered time domain features, which are enough for data sets classification. The statistical features are mean value, standard deviation, and skewness value. The expressions are given as follows [21]:

$$
\text { Mean Value }=\frac{1}{N} \sum_{i=1}^{N} x_{i},
$$

$$
\begin{gathered}
\text { Standard Deviation }=\sqrt{\frac{1}{N} \sum_{i=1}^{N}\left(x_{i}-\mu\right)^{2}}, \\
\text { Skewness Value }=\frac{1}{N} \sum_{i=1}^{N}\left(\frac{\left|x_{i}-\mu\right|}{\sigma}\right)^{3} .
\end{gathered}
$$

Where $\mathrm{N}$ is the total number of frequencies, $\mathrm{xi}$ denotes each sampling points, $\mu$ is the mean value of sampling data, $\sigma$ denotes the standard deviation of the sampling data. Figure 7 shows the distribution of two classes in their feature space.

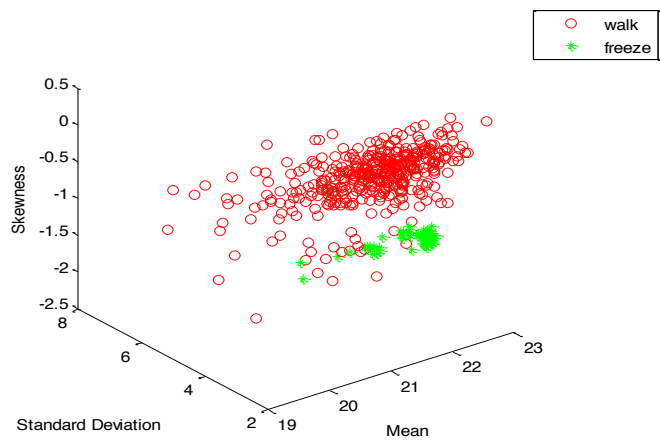

Figure 7 - Example of walking and freezing of gait presented in their feature space.

The classification of walking and freezing of gait may be confusing by considering the three features. Thus we choose the best classification performers to measure the performance of the proposed method as illustrated in Figure 8. Considering the best performers such as standard deviation and skewness, the SVM provides an error rate of $1 \%$.

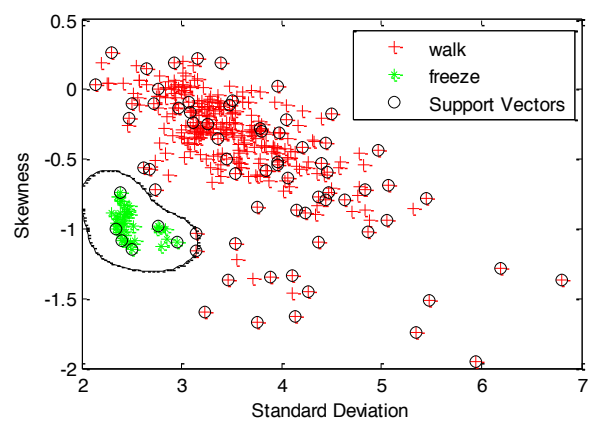

Figure 8 - Classification of walking and freezing of gait using SVM considering standard deviation and skewness 


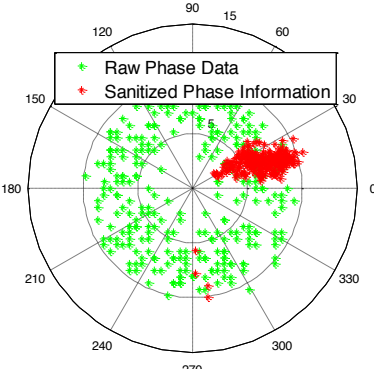

(a). Walking

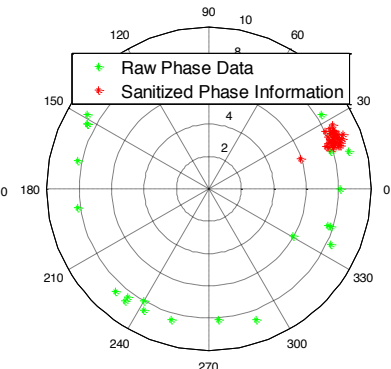

(b). Freezing of gait

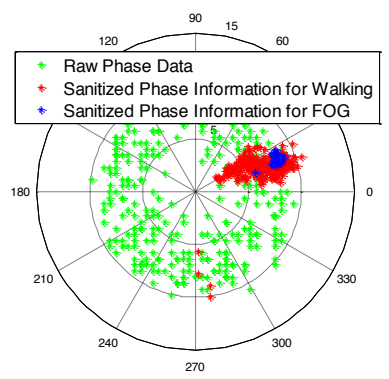

(c). Walking and freezing of gait

Figure 9 - Raw phase data and sanitized phase information obtained for (a).walking (b).freezing of gait (c).walking and freezing of gait

As discussed above, the raw phase information is random and cannot be used for applications. Hence, using linear transformation, useful (sanitized) phase information is obtained for detecting freezing episodes in the PD patient as depicted in Figure 9. The sanitized data vary significantly due to the person's walking across the room, as shown in Figure 9(a). On the other hand, a cluster of phase information in Figure 9(b) is static due to the subject experiencing FOG episode. Figure 9(c) presents the sanitized phase information for walking and freezing of gait. It is worth mentioning that they overlap between $0^{\circ}$ and $30^{\circ}$; therefore it is necessary to classify them (as shown in Figure 8).

\section{B. Scenario II: FOG detection in multiple patients}

In this scenario, we examine the measured data for multiple subjects in two spatially separated areas. The experiment was performed according to the subject data given in Table-2. A leaky wave cable of 3-meter-long is used as the receiving antenna as illustrated in Figure 2(b). Two dedicated routers were deployed for the two rooms operating at $2.41 \mathrm{GHz}$ and $2.45 \mathrm{GHz}$ to avoid co-channel interference.

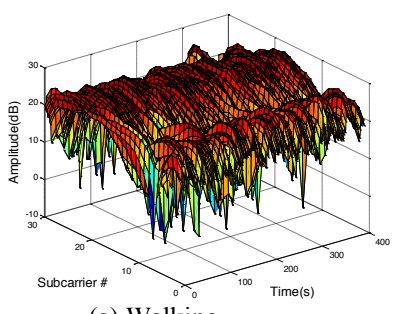

(a).Walking

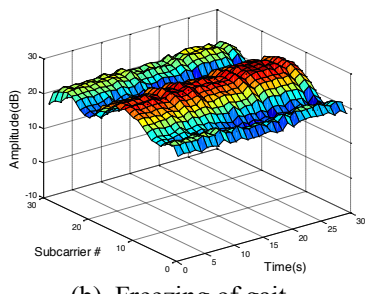

(b). Freezing of gait
Figure 10 - Raw CFR traces obtained for person in large conference room (a). Walking for 400 seconds

(b). Experiencing freezing episodes for 30 seconds.
We first analyze the data obtained for subject in the large conference room. Figure 10 shows the raw channel information data obtained for 430 seconds. Figure 10(a) indicates the raw channel information data for person walking inside the premises for 400 seconds with a speed of 1.31 meters/second while Figure 10(b) shows the raw traces of freezing of gait for 30 seconds.

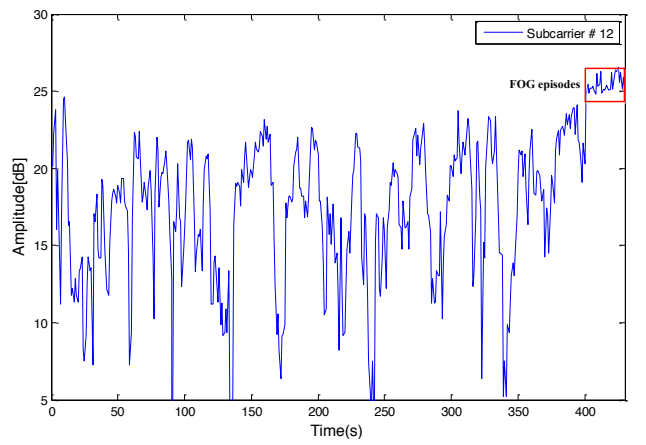

Figure 11 - Time history of amplitude information when experiment was conducted in large conference room.

A similar approach discussed in the previous section is employed. Subcarrier \# 12 is used to examine the time history of person's gait and extract the freezing episodes considering the variances of amplitude information as in Figure 11. It is not possible to differentiate the freezing episodes from normal gait (walking). Thus the SVM is used for detecting FOG episodes of single person.

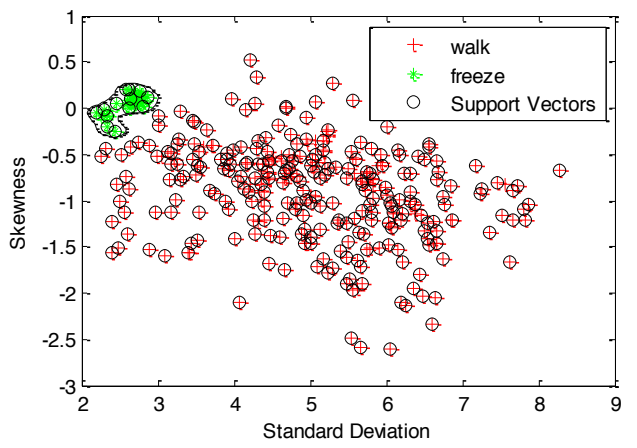

Figure 12 - Classification of walking and FOG episodes using SVM considering standard deviation and skewness

Figure 12 shows the classification of two data sets considering standard deviation and skewness. A cluster in green indicates the dataset obtained for FOG episodes and the scattering data in red shows walk across the room. For classification in this case, the error rate of $0 \%$ is achieved.

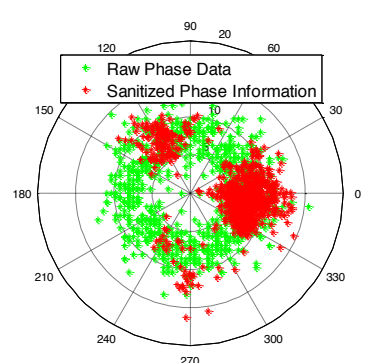

(a).Walking

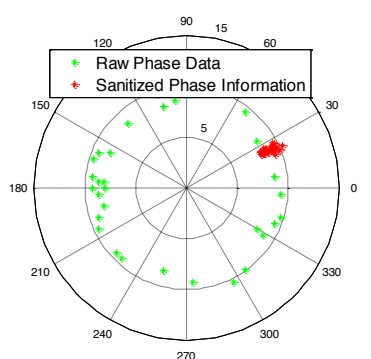

(b). Freezing of gait 


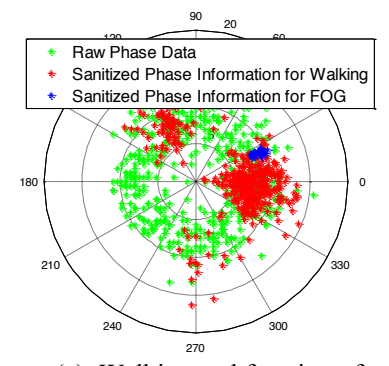

(c). Walking and freezing of gait

Figure 13 - Raw CSI phase data and sanitized phase information obtained from experiment in large conference room (a).walking (b). FOG episodes

(c). walking and FOG of gait

Figure 13(a) shows the sanitized phase information of subject walking in large conference room. The data in this case is dispersed indicating that the person was walking. While sanitized phase information in Figure 13(b) seems to be stationary at one place, which clearly show FOG episode. The phase data of freezing of gait is concentrated between $15^{\circ}$ and $30^{\circ}$, which intersects with some phase data of walking, as illustrated in Figure 13(c). SVM is applied to distinguish the two.

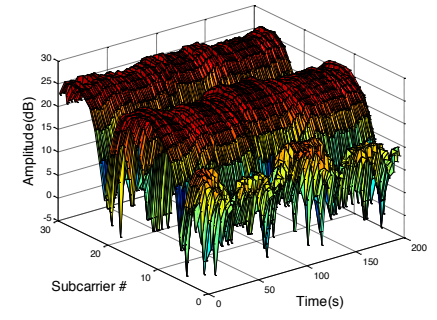

(a).Walking

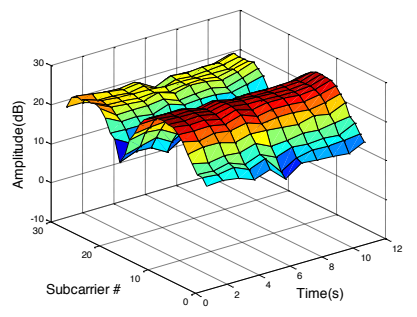

(b). Freezing of gait
Figure 14 - (a). Raw CFR traces for person walking inside the sitting room for 200 seconds. (b). Raw CFR data for subject experiencing FOG for 11 seconds.

Next, data recorded for the patient in the sitting room is analyzed. Figure 14(a) shows that the subject walks for 200 seconds at a speed of 0.89 meters/second. The raw channel information amplitude data in Figure 14(b) is obtained when FOG episodes were observed for a period of 11 seconds.

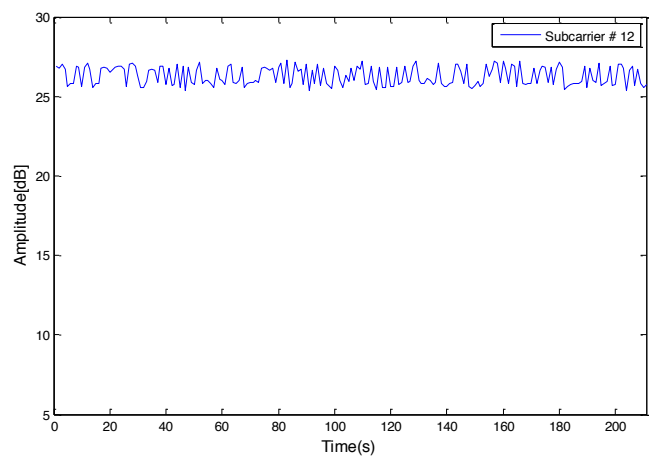

Figure 15 - Time history of amplitude information when experiment was conducted in sitting conference room.

Figure 15 shows that the experiment was conducted for 211 seconds. The variance of amplitude for subcarrier \#12 against the time history does not give adequate information for the
FOG detection. The primary reason is that the person's pace was very slow resulting in a low level of variances. Hence, in order to detect the FOG episodes, the SVM is needed.

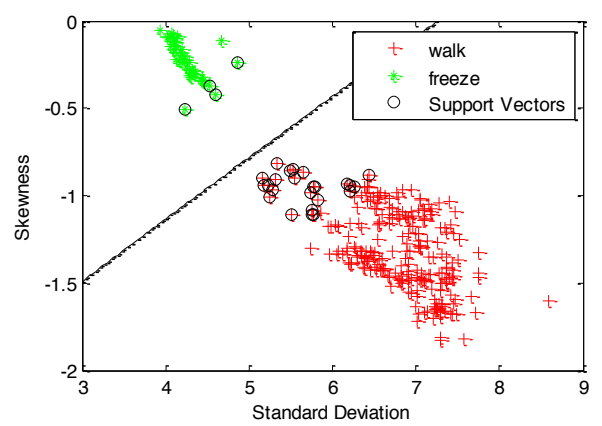

Figure 16 - Classification of walking and FOG episodes using SVM considering standard deviation and skewness (sitting room)

Figure 16 shows the data classification. The data in green illustrates FOG episodes and the one in red is for the person walking inside the sitting room. The data for sitting room also gives an error rate of $0 \%$. Considering the sanitized phase information for the experimental data in sitting room, Figure 17(a) shows the sanitized phase information to be dispersed due to walking as compared to the data in Figure 17(b), where the stationary cluster shows the FOG episodes. To compare the two phase data, the sanitized phase information for walking and freezing of gait is shown in Figure 17(c). It can be observed that the two states are well distinctive in sitting room.

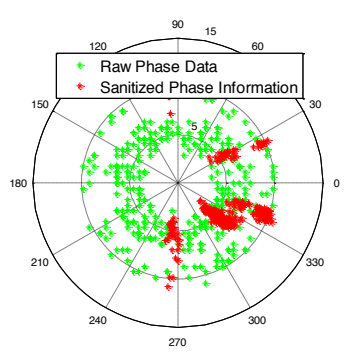

(a). Walking

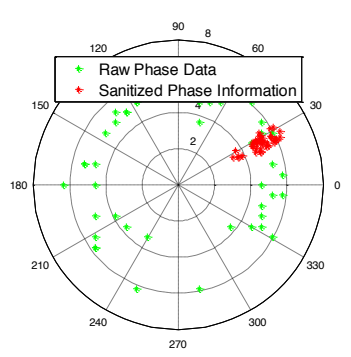

(b). Freezing of gait

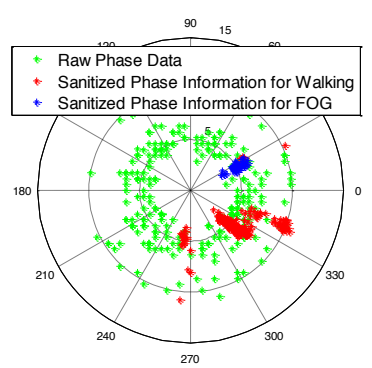

(c). Walking and freezing of gait

Figure 17 - Raw phase data and sanitized phase information obtained from experiment in sitting room (a). Walking (b). FOG episodes (c). walking and FOG of gait

It is also interesting to note that it might be feasible to establish the mathematical relationship between the dynamic changes of signals and the rate of change of lengths for reflected paths. It is found by researchers [22] that the energy of channel information is the sum of a series of trigonometric functions, and the frequencies of the trigonometric functions 
are related to the rate of change of the lengths for reflected paths caused by the movement of human body. The speed extraction approach is based on the time-frequency analysis. It is also worth mentioning that this model is applicable when the subject walks not parallel to the transmitters and receivers. In this work, accurate classification results have been achieved and only moving and freezing behaviors are considered. The speed model would be considered in future extensions of this study.

\section{CONCLUSION}

The PD is a neurodegenerative disorder and its progressive nature is demonstrated by a range of motor and non-motor symptoms. Non-motor issues are common among the PD patients resulting in certain abnormalities including neurobehavioral changes. In case of the FOG, the secondary reasons such as fall and associated cognitive impairments can be diagnosed clinically to follow alternate therapies.

In this work, we have presented a non-invasive method to detect the FOG episodes by exploring the wireless channel characteristics and using a single LWC to support spatially dispersed multiple PD patients, which is different from traditional wearable channel characterization methods [23]. The LWC accommodates unique operating frequency for each subject and the granularity of time scale can be changed. The close spacing of carrier frequency also helps in accommodating more patients over wide range of distance. The method employs wireless channel information and SVM to detect the FOG conditions. The data acquisition provides a consolidated packet of data per patient. The seamless communication link maintains a high-fidelity factor between monitoring system and the patients. The validity and accuracy of the proposed method is established through measurements. It is evident from the presented results that a particular FOG condition observed in a specific sub-channel for shorter duration can help in looking closely to the conditions prevailing. The method has also yielded high accuracy level of $99 \%$ in various measurement scenarios.

Further improvement of the monitoring systems includes real-time alerts to the attendant who can provide a stimulus to the patient and avoid risks of falling.

\section{REFERENCES}

[1]. W. Muangpaisan, A. Mathews, H. Hori, and D. Seidel, "A systematic review of the worldwide prevalence and incidence of Parkinson's disease," J. Med. Assoc. Thailand, vol. 94, pp. 749-755, 2011.

[2]. J. Jankovic, "Parkinson's disease: Clinical features and diagnosis," J.Neurol. Neurosurg. Psychiatry, vol. 79, no. 4, pp. 368-376, 2008.

[3]. T. Hashimoto, "Speculation on the responsible sites and pathophysiology of freezing of gait," Parkinsonism Rel. Disord., vol. 12, pp. S55-S62, 2006.

[4]. M. Mancini, K. C. Priest, J. G. Nutt, and F. B. Horak, "Quantifying freezing of gait in Parkinson's disease during the instrumented timed up and go test," in Conf Proc IEEE Eng Med Biol Soc 2012, 2012, pp. 1198-1201.

[5]. S. T. Moore, H. G. MacDougall, and W. G. Ondo, "Ambulatory monitoring of freezing of gait in Parkinson's disease,"J Neuroscience Methods., vol. 167, pp. 340-348, 2008.

[6]. M. Bachlin, M. Plotnik, D. Roggen, I. Maidan, J. M. Hausdorff, N. Giladi, and G. Troster, "Wearable assistant for Parkinson's disease patients with the freezing of gait symptom,"'Information Technology in Biomedicine, IEEE Transactions on, vol. 14, no. 2, pp. 436-446, 2010.

[7]. S. Mazilu, M. Hardegger, Z. Zhu, D. Roggen, G. Troster, M. Plotnik, and
J. M. Hausdorff, "Online detection of freezing of gait with smartphones and machine learning techniques," in 6th Intern. Conf. on Pervasive Computing Technologies for Healthcare (PervasiveHealth) and Workshops, 2012.

[8]. E. E. Tripoliti, A. T. Tzallas, M. G. Tsipouras, G. Rigas, P. Bougia, M. Leontiou, S. Konitsiotis, M. Chondrogiorgi, S. Tsouli, and D. I. Fotiadis, "Automatic detection of freezing of gait events in patients. with Parkinson's disease,"Comput Methods Programs Biomed, vol. 110, pp. 12-26, 2013.

[9]. Mazilu, Sinziana, et al. "Prediction of Freezing of Gait in Parkinson's from physiological wearables: an exploratory study." IEEE journal of biomedical and health informatics 19.6 (2015): 1843-1854.

[10]. Q. Pu, S. Gupta, S. Gollakota, and S. Patel, "Whole-home gesture recognition using wireless signals," in Proc. ACM MobiCom, 2013, pp. 27-38.

[11]. C. $\mathrm{Xu}$ et al., "SCPL: Indoor device-free multi-subject counting and localization using radio signal strength," in Proc. ACM IPSN, 2013, pp. 79-90.

[12]. Yang, Xiaodong, et al. "Wandering Pattern Sensing at S-Band." IEEE Journal of Biomedical and Health Informatics (2017).

[13]. D. Halperin, W. Hu, A. Sheth, and D. Wetherall, "Predictable 802.11 packet delivery from wireless channel measurements," in Proc. ACM SIGCOMM, 2010, pp. 159-170.

[14]. C. Cortes and V. Vapnik, "Support-vector networks," Mach. Learn., Vol. 20, no. 3, pp. 273-297, 1995.

[15]. S. R. Gunn, "Support vector machines for classification and regression,"Faculty Eng., Sci. Mathemat. School Electron. Comput. Sci., Univ. Southampton, Southampton, U.K., Tech. Rep. 256459, May 1998.

[16]. X. Wang, L. Gao and S. Mao, "CSI Phase Fingerprinting for Indoor Localization With a Deep Learning Approach," in IEEE Internet of Things Journal, vol. 3, no. 6, pp. 1113-1123, Dec. 2016.

[17]. Masayuki Nakamura, Hideaki Takagi, Kiyoshi Einaga, Toshiyuki Nishikawa, Naoshi Moriyama and Katsumi Wasaki, "Development of a $300 \mathrm{~m} 2.4 \mathrm{GHz}$ frequency band leaky coaxial cable for wireless network access," 2008 IEEE Radio and Wireless Symposium, Orlando, FL, 2008, pp. $687-690$.

[18]. K. Inomata, W. Tsujita and T. Hirai, "Two-frequency surveillance technique for intrusion-detection sensor with Leaky Coaxial Cables," 2014 IEEE Sensors Applications Symposium (SAS), Queenstown, 2014, pp. 103-106.

[19]. IEC 61196-4, International Standard, 2015.

[20]. S. Maheshwari and A. K. Tiwari, "Walking parameters estimation through channel state information preliminary results," Signal Processing and Communication Systems (ICSPCS), 2015 9th International Conference on, Cairns, QLD, 2015, pp. 1-8.

[21]. Bing Song, Hongbo Shi, "Temporal-Spatial Global Locality Projections for Multimode Process Monitoring", IEEE Access, vol. 6, pp. 9740-9749, 2018, ISSN 2169-3536.

[22]. Wei Wang, Alex X. Liu, Muhammad Shahzad, Kang Ling, Sanglu Lu, "Understanding and Modeling of WiFi Signal Based Human Activity Recognition," Proceedings of the 21st Annual International Conference on Mobile Computing and Networking, Paris, France - September 07 - 11, 2015, pp. 65-76.

[23]. Masood Ur-Rehman, Nabeel A. Malik, Xiaodong Yang, Qammer H. Abbasi, Zhiya Zhang, Nan Zhao, "A Low Profile Antenna for Millimeter-Wave Body-Centric Applications," IEEE Transactions on Antennas and Propagation, Vol. 65, No. 12, pp. 6329-6337, December, 2017 